\title{
Steric Dependence of Chirality Effect in Surface-Mediated Peptide Assemblies Identified with Scanning Tunneling Microscopy
}

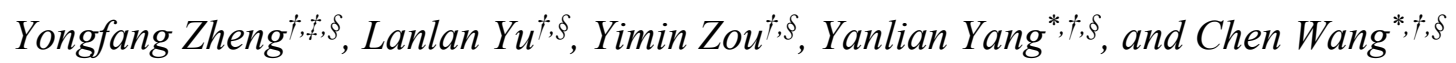

${ }^{\dagger}$ CAS Key Laboratory of Biological Effects of Nanomaterials and Nanosafety, CAS

Key Laboratory of Standardization and Measurement for Nanotechnology, CAS Center

for Excellence in Nanoscience, National Center for Nanoscience and Technology, Beijing 100190, P. R. China

tDepartment of Chemistry, Tsinghua University, Beijing 100084, P. R. China

${ }^{\S}$ University of Chinese Academy of Sciences, Beijing 100049, P. R. China

*Email: yangyl@nanoctr.cn, Fax: +86 10 62656765, Tel: +86 1082545559

*Email: wangch@nanoctr.cn, Fax: +86 10 62656765, Tel: +86 1082545561 


\section{Supporting information}

\section{Materials and methods}

Sample Preparation: Peptides were purchased from Bankpeptide Biological Technology Co., Ltd. 4, 4-bipyridyl (4Bpy) was purchased from Sigma-Aldrich Co. Ltd. All the materials were used without further purification.

Lyophilized powders of peptides were dissolved in $50 \mathrm{uL}$ of dimethyl sulfoxide (DMSO) first and then dilute it with Milli-Q water to reach a concentration of $0.3-0.4 \mathrm{mg} / \mathrm{mL}$. 4Bpy is dissolved in Milli-Q water to reach a concentration of $1 \mathrm{mg} / \mathrm{mL}$. Mix $5 \mu \mathrm{L}$ of peptide solution with $1500 \mu \mathrm{L}$ of 4Bpy solution. $10 \mu \mathrm{L}$ of the mixed solution was dropped on freshly cleaved HOPG surface. Blow away the remained solution with nitrogen gas after incubation for 20 minutes.

The STM experiments were carried out after the solvent was dried in ambient conditions. Previous STM experiments showed high resolution structural analysis of peptide assemblies can be studied using ethanol ${ }^{1}$, Milli-Q water $^{2}$, or acetonitrile ${ }^{3}$ as solvents in sample preparation and the obtained assembly characteristics, such as inter-peptide spacings, are comparable. It is therefore plausible to consider that solvents have little effects on the observed structural characteristics of assembly pattern in this work.

Concentration is an important factor that may affect surface-bound co-assembly structures as the result of energetics and kinetics effects ${ }^{4-7}$. For the concentration dependence of the assembly structures in this study, we indeed observed that the reduced concentrations led to apparent phaseseparated domains of co-assembly of Poly7Q/4Bpy, pristine peptides and 4Bpy. Such variation in the observed domain dimensions is a reflection of overall concentrations and ratio of peptide and 4Bpy, and will not affect the measured structural characteristics reported in the manuscript.

The choice of HOPG is due to its well-known chemical inertness and atomically flat surface which are ideal for STM observations in ambient conditions. Replacing HOPG with other surfaces (such as metallic single crystal surfaces) will inevitably affect the commensurability between the surface atomic lattice and periodicity of peptide assembly structures, as well as peptide-substrate and peptide-peptide interactions. The pursuit of surface-dependence of fine structures of peptide assemblies could greatly enrich the molecular understanding of the bio-nano interfaces that are central to the nanobiotechnologies.

STM Experiments: STM observations were carried out with a Nanoscope IIIa scanning probe microscopy system (Bruker, U.S.A), using mechanically formed Pt/Ir (80/20) tips under ambient conditions. The tunneling conditions are shown in the corresponding figure captions.

FTIR measurements: The infrared spectra were recorded using a Perkin Elmer Spectrum One FTIR Spectrometer (Waltham, Massachusetts, USA). Dried film samples were prepared by dropping $20 \mu \mathrm{L}$ of the sample solution onto $\mathrm{CaF}_{2}$ plates. All spectra were scanned over the range of $2000-1000 \mathrm{~cm}^{-1}$ at a resolution of $4 \mathrm{~cm}^{-1}$.

STM image calibration: All the STM images in this work were calibrated by comparing images of peptide/4Bpy co-assembly with atomically resolved atomic lattice of HOPG which has been used as standard sample for STM calibration. Herein, the calibration of Poly7Q was taken as an example. As shown in Figure.S1, high-resolution STM image of Poly7Q assembly was obtained first with $I$ at $299.1 \mathrm{pA}$ and $V$ at $699.8 \mathrm{mV}$. Then changing the voltage and current to $I=1000.0 \mathrm{pA}$ and $V=45.0 \mathrm{mV}$ in situ, to obtain the atomic lattice of HOPG surface. The unit cell parameters of HOPG are known precisely ( $\left.a=2.46 \AA, b=2.46 \AA, \alpha=120^{\circ}\right)$ and the lengths of the stably deposited 
peptide chains can be calibrated with these values.

Statistical Method: As shown in Figure S2, the lengths of the stably deposited peptide chains were measured using the Nanoscope software (Bruker) and calibrated. The measured lengths of more than 100 peptide chains have been randomly obtained from independently recorded STM images, which we consider as a balanced reflection of the length distribution. Table S1 and Table S2 present the counts of the measured peptide lengths within the corresponding length ranges. The increment of the statistical histograms is $0.325 \mathrm{~nm}$, which is the distance of two neighboring amino acid residues in parallel $\beta$-sheet structures. The statistical histograms were fitted by Gaussian distribution.

Table S1. Statistical length distributions of Poly7Q and its seven mutant peptides.

\begin{tabular}{|c|c|c|c|c|c|c|c|c|c|}
\hline $\begin{array}{l}\text { range of peptide } \\
\qquad \text { length }(\mathbf{n m})^{a}\end{array}$ & $\begin{array}{l}\text { number of } \\
\text { residues }^{b}\end{array}$ & $\begin{array}{c}\text { counts }^{c} \text { of } \\
\text { Poly7Q }\end{array}$ & $\begin{array}{c}\text { counts }^{c} \text { of } \\
\text { Polyq7 }\end{array}$ & $\begin{array}{c}\text { counts }^{c} \text { of } \\
\text { Polyq6 }\end{array}$ & $\begin{array}{c}\text { counts }^{c} \text { of } \\
\text { Polyq5 }\end{array}$ & $\begin{array}{c}\text { counts }^{c} \text { of } \\
\text { Polyq4 }\end{array}$ & $\begin{array}{c}\text { counts }^{c} \text { of } \\
\text { Polyq3 }\end{array}$ & $\begin{array}{c}\text { counts }^{c} \text { of } \\
\text { Polyq2 }\end{array}$ & $\begin{array}{c}\operatorname{counts}^{c} \text { of } \\
\text { Polyq1 }\end{array}$ \\
\hline $2.7625-3.0875$ & $8.5-9.5$ & 0 & 0 & 0 & 5 & 0 & 1 & 0 & 0 \\
\hline $3.0875-3.4125$ & $9.5-10.5$ & 0 & 0 & 6 & 21 & 17 & 9 & 0 & 0 \\
\hline $3.4125-3.7375$ & $10.5-11.5$ & 0 & 0 & 33 & 51 & 51 & 48 & 15 & 13 \\
\hline $3.7375-4.0625$ & $11.5-12.5$ & 0 & 0 & 51 & 31 & 32 & 43 & 81 & 49 \\
\hline $4.0625-4.3875$ & $12.5-13.5$ & 8 & 16 & 28 & 0 & 0 & 2 & 26 & 39 \\
\hline $4.3875-4.7125$ & $13.5-14.5$ & 71 & 79 & 6 & 0 & 0 & 0 & 1 & 0 \\
\hline $4.7125-5.0375$ & $14.5-15.5$ & 22 & 7 & 0 & 0 & 0 & 0 & 0 & 0 \\
\hline
\end{tabular}

${ }^{a}$ Range of peptide length is divided according to the minimum and the maximum lengths of peptides measured from STM images in increments of $0.325 \mathrm{~nm}$ (the separation between two neighboring residues in parallel beta-sheet structures). ${ }^{b}$ Number of residues is calculated by dividing the peptide length by $0.325 \mathrm{~nm}$. ${ }^{c}$ Counts is the number of peptide lengths within the related range.

Table S2. Statistical length distributions of Poly7N, Poly7E, Poly7D, Poly7A and their mutant peptides.

\begin{tabular}{|c|c|c|c|c|c|c|c|c|c|}
\hline $\begin{array}{l}\text { range of peptide } \\
\text { length }(\mathbf{n m})^{\mathrm{a}}\end{array}$ & $\begin{array}{l}\text { number of } \\
\text { residues }^{b}\end{array}$ & $\begin{array}{c}\text { counts }^{c} \text { of } \\
\text { Poly7N }\end{array}$ & $\begin{array}{c}\text { counts }^{c} \text { of } \\
\text { Polyn4 }\end{array}$ & $\begin{array}{c}\text { counts }^{c} \text { of } \\
\text { Poly7E }\end{array}$ & $\begin{array}{c}\text { counts }^{c} \text { of } \\
\text { Polye4 }\end{array}$ & $\begin{array}{c}\text { counts }^{c} \text { of } \\
\text { Poly7D }\end{array}$ & $\begin{array}{c}\text { counts }^{c} \text { of } \\
\text { Polyd4 }\end{array}$ & $\begin{array}{c}\text { counts }^{c} \text { of } \\
\text { Poly7A }\end{array}$ & $\begin{array}{c}\text { counts }^{c} \text { of } \\
\text { Polya4 }\end{array}$ \\
\hline 2.7625-3.0875 & $8.5-9.5$ & 0 & 0 & 0 & 22 & 0 & 1 & 0 & 0 \\
\hline 3.0875-3.4125 & $9.5-10.5$ & 0 & 0 & 0 & 92 & 0 & 5 & 0 & 0 \\
\hline $3.4125-3.7375$ & $10.5-11.5$ & 0 & 0 & 0 & 74 & 0 & 47 & 0 & 0 \\
\hline $3.7375-4.0625$ & $11.5-12.5$ & 4 & 31 & 11 & 19 & 3 & 70 & 16 & 6 \\
\hline $4.0625-4.3875$ & $12.5-13.5$ & 28 & 68 & 80 & 0 & 69 & 102 & 138 & 70 \\
\hline $4.3875-4.7125$ & $13.5-14.5$ & 74 & 7 & 80 & 0 & 63 & 34 & 117 & 28 \\
\hline $4.7125-5.0375$ & $14.5-15.5$ & 5 & 0 & 28 & 0 & 14 & 0 & 6 & 0 \\
\hline 5.0375-5.3625 & $15.5-16.5$ & 0 & 0 & 4 & 0 & 0 & 0 & 0 & 0 \\
\hline
\end{tabular}

${ }^{a}$ Range of peptide length is divided according to the minimum and the maximum lengths of peptides measured from STM images in increments of $0.325 \mathrm{~nm}$ (the separation between two neighboring residues in parallel beta-sheet structures). ${ }^{b}$ Number of residues is calculated by dividing the peptide length by $0.325 \mathrm{~nm}$. ${ }^{c}$ Counts is the number of peptide length within the related range. 


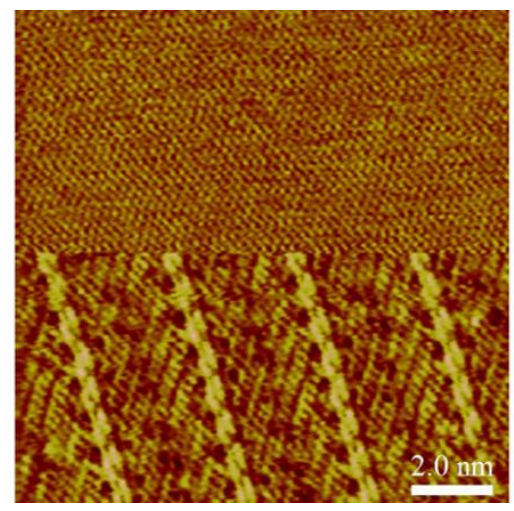

Figure S1. STM image for poly7Q/4Bpy co-assembly and HOPG

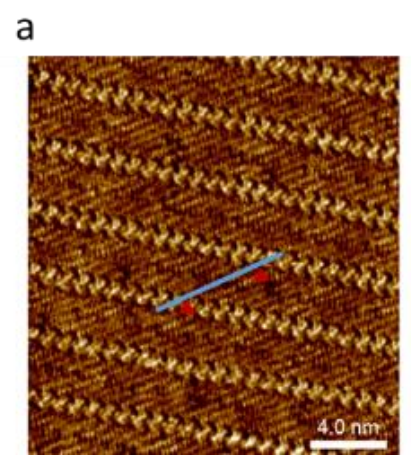

b

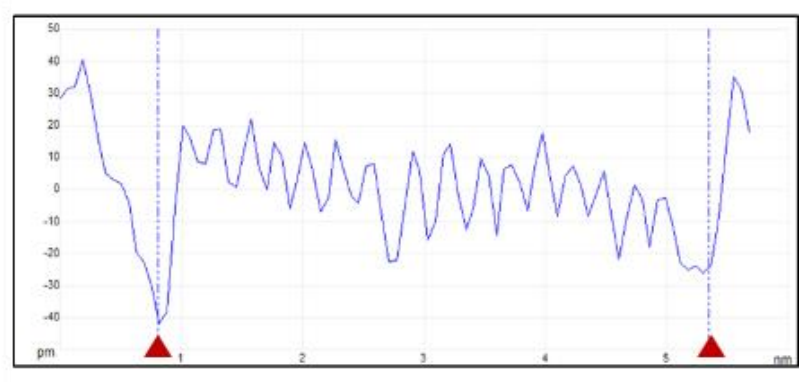

Figure S2. a. The STM image of polyq3/4Bpy co-assembly structure. The distance between the two red triangles corresponds to the length of peptide chain. $b$. The sectional profile corresponding to the blue line in (a).

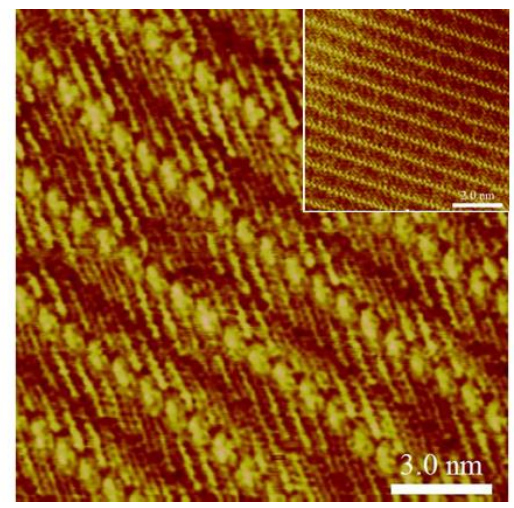

Figure S3. High-resolution STM image of polyq1/4Bpy co-assembly structure. The Inset is a large scale STM image of polyq1/4Bpy. Tunneling conditions: $I=399.8 \mathrm{pA}, V=649.7 \mathrm{mV}$. 


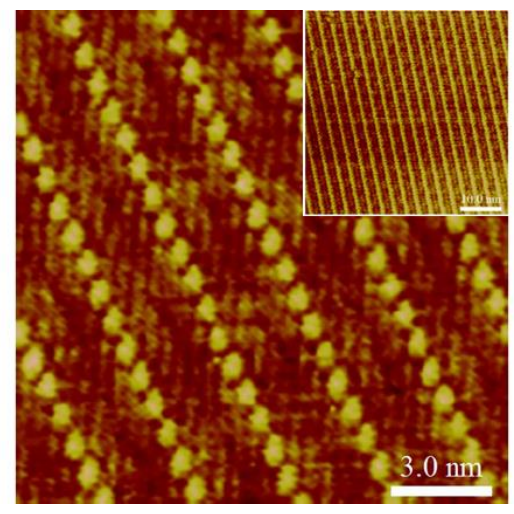

Figure S4. High-resolution STM image of polyq2/4Bpy co-assembly structure. The inset is a large scale STM image of polyq2/4Bpy assembly. Tunneling conditions: $I=299.8 \mathrm{pA}, V=699.8 \mathrm{mV}$.

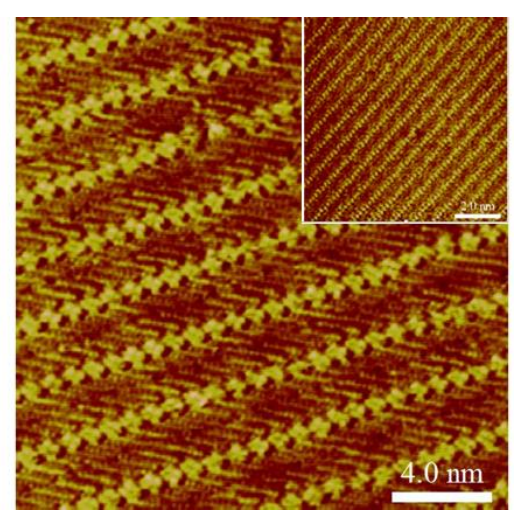

Figure S5. High-resolution STM image of polyq4/4Bpy co-assembly structure. The inset is a large scale STM image of polyq4/4Bpy. Tunneling conditions: $I=296.0 \mathrm{pA}, V=698.9 \mathrm{mV}$.

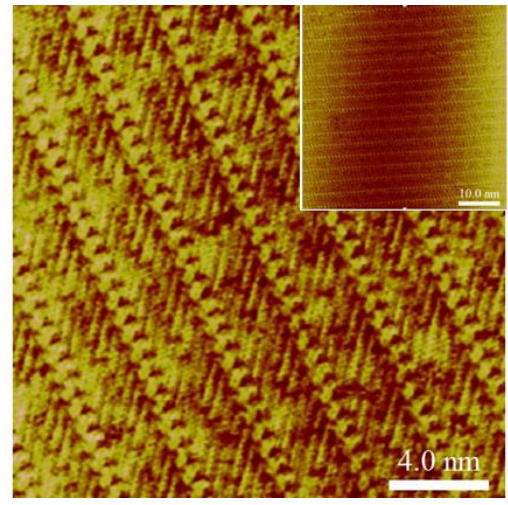

Figure S6. High-resolution STM image of polyq5/4Bpy co-assembly structure. The inset is a large scale STM image of polyq5/4Bpy. Tunneling conditions: $I=299.1 \mathrm{pA}, V=699.8 \mathrm{mV}$. 


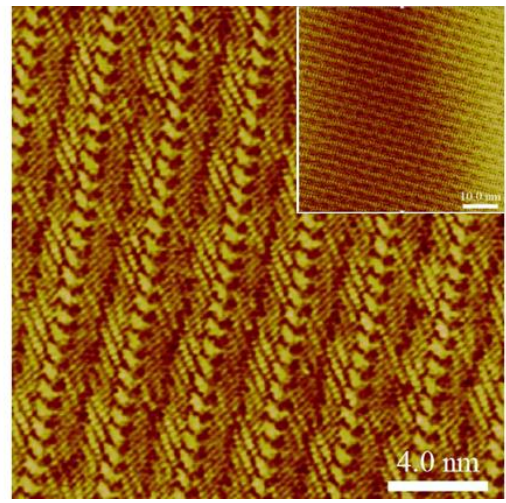

Figure S7. High-resolution STM image of polyq6/4Bpy co-assembly structure. The inset is a large scale STM image of polyq6/4Bpy. Tunneling conditions: $I=320.0 \mathrm{pA}, V=698.9 \mathrm{mV}$.

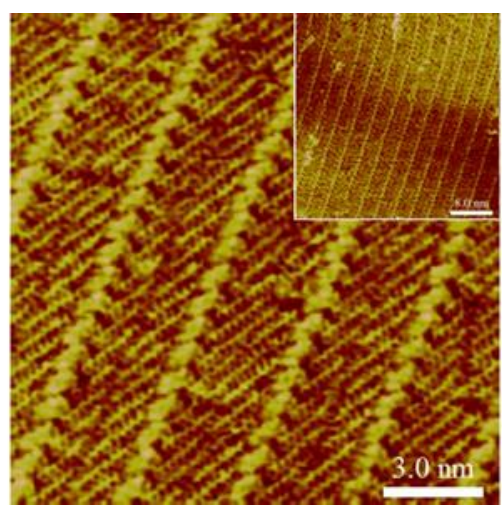

Figure S8. High-resolution STM image of polyq7/4Bpy co-assembly structure. The inset is a large scale STM image of polyq7/4Bpy. Tunneling conditions: $I=320.0 \mathrm{pA}, V=698.9 \mathrm{mV}$.

(a)

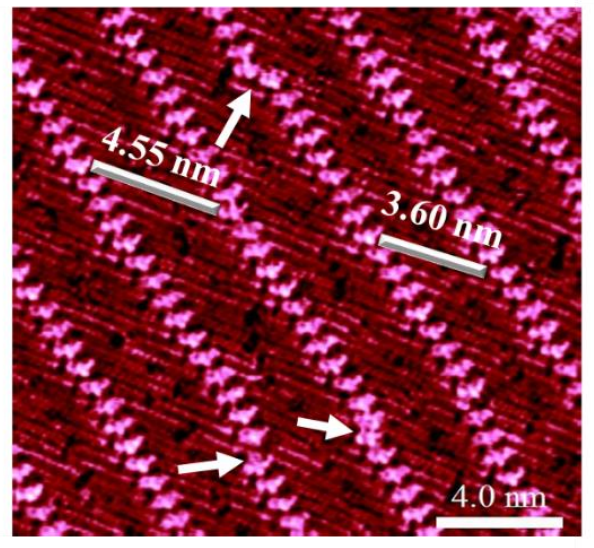

(b)

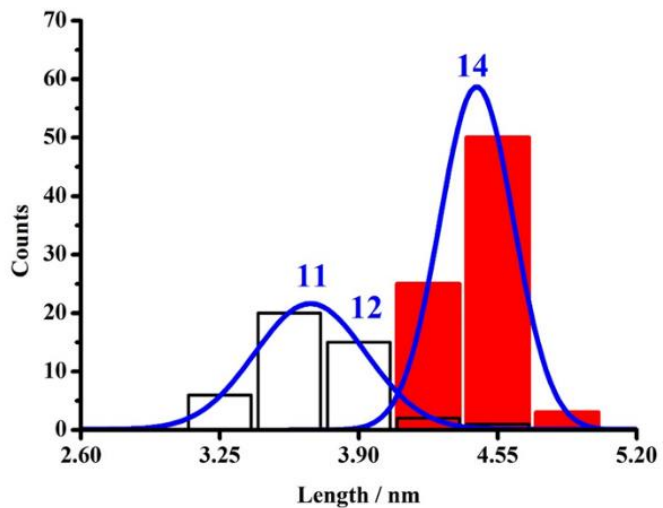

Figure S9. a. High-resolution STM image of Polyq7/4Bpy co-assembly structure. The white lines show us the long and short peptide chains involved in assembly and the white arrows indicate the turning points in 4Bpy strand. Tunneling conditions: $I=296.0 \mathrm{pA}, V=699.8 \mathrm{mV}$. b. The statistical histogram with Gaussian fitting of the length distribution of peptide chains in the small marginal assembly scope of Polyq7. 


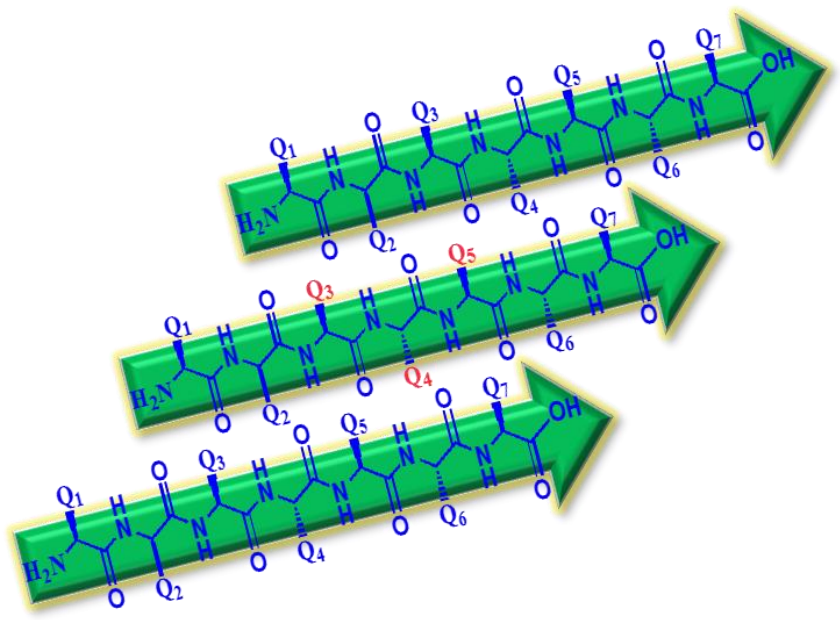

Figure S10. Model of Poly7Q peptide in assembly.

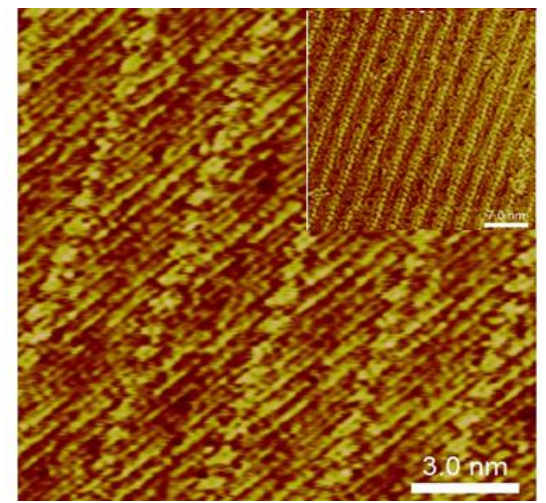

Figure S11. High-resolution STM image of poly7N/4Bpy co-assembly structure. The inset is a large scale STM image of poly7N/4Bpy. Tunneling conditions: $I=299.0 \mathrm{pA}, V=698.9 \mathrm{mV}$.

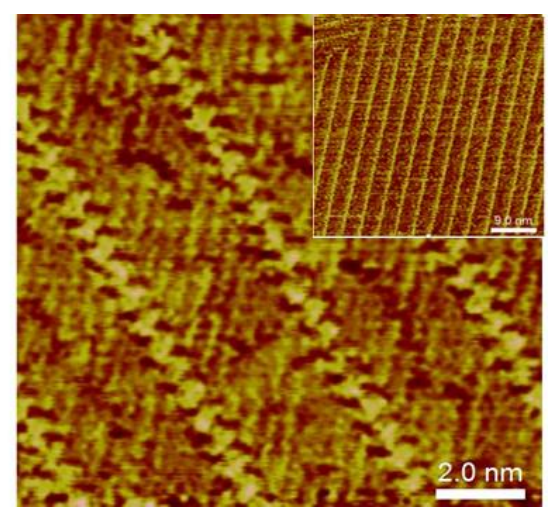

Figure S12. High-resolution STM image of polyn4/4Bpy co-assembly structure. The inset is a large scale STM image of polyn4/4Bpy. Tunneling conditions: $I=299.0 \mathrm{pA}, V=698.9 \mathrm{mV}$. 


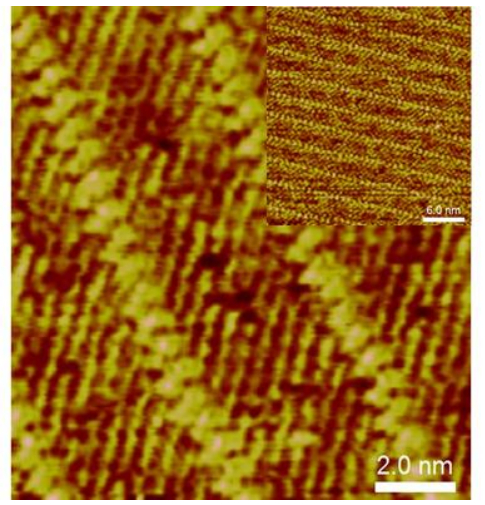

Figure S13. High-resolution STM image of poly7E/4Bpy co-assembly structure. The inset is a large scale STM image of poly7E/4Bpy. Tunneling conditions: $I=320.0 \mathrm{pA}, V=698.9 \mathrm{mV}$.

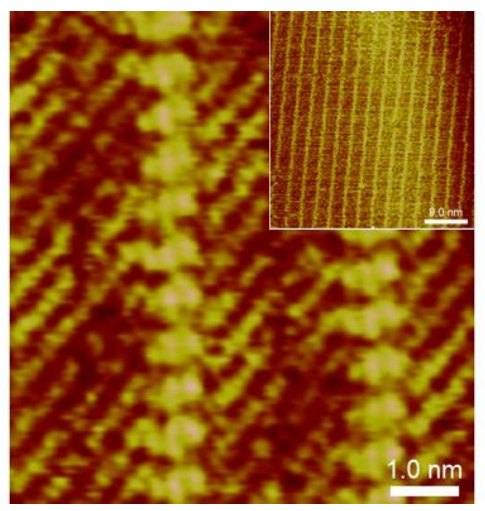

Figure S14. High-resolution STM image of polye4/4Bpy co-assembly structure. The inset is a large scale STM image of polye4/4Bpy. Tunneling conditions: $I=299.0 \mathrm{pA}, V=698.9 \mathrm{mV}$.

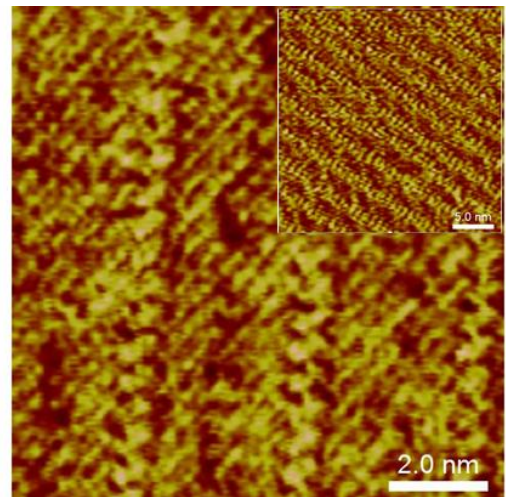

Figure S15. High-resolution STM image of poly7D/4Bpy co-assembly structure. The inset is a large scale STM image of poly7D/4Bpy. Tunneling conditions: $I=320.0 \mathrm{pA}, V=698.9 \mathrm{mV}$. 


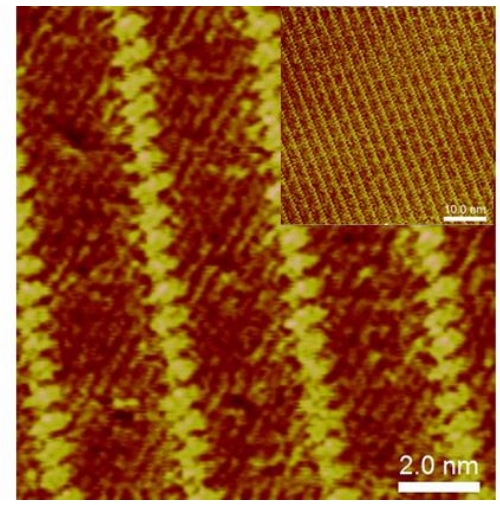

Figure S16. High-resolution STM image of polyd4/4Bpy co-assembly structure. The inset is a large scale STM image of polyd4/4Bpy. Tunneling conditions: $I=296.0 \mathrm{pA}, V=698.9 \mathrm{mV}$.

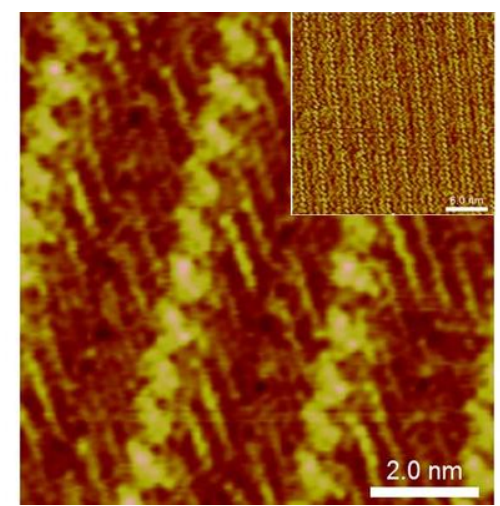

Figure S17. High-resolution STM image of polya4/4Bpy co-assembly structure. The inset is a large scale STM image of polya4/4Bpy. Tunneling conditions: $I=299.0 \mathrm{pA}, V=698.9 \mathrm{mV}$.

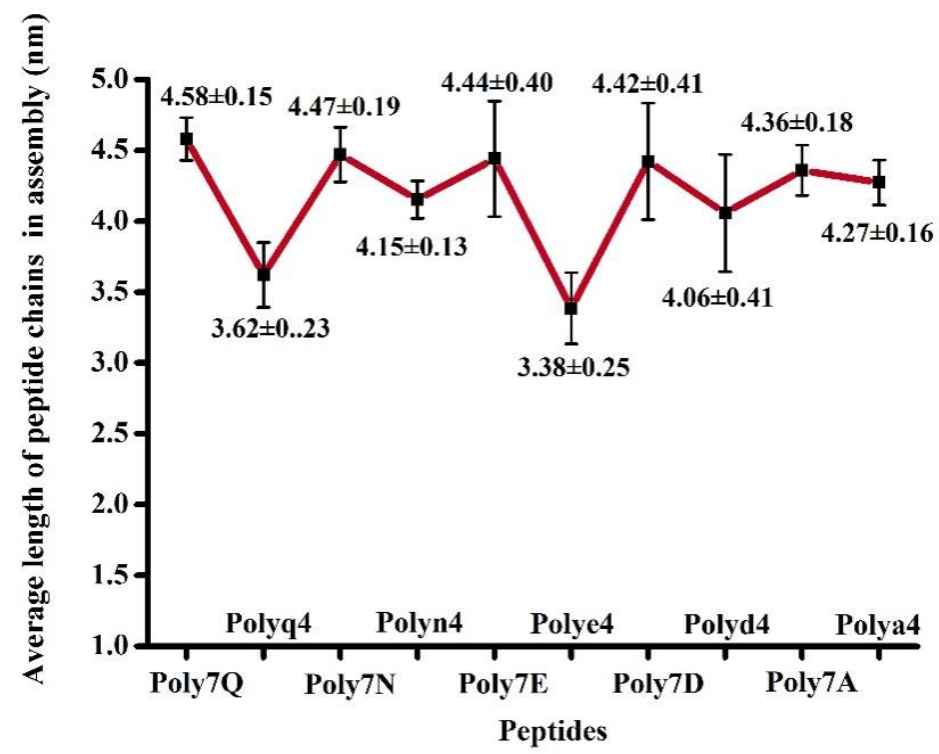

Figure S18. The average lengths of peptide chains in the assemblies of Poly7Q, Poly7N, Poly7E, Poly7D, Poly7A and their mutants. 
(a)

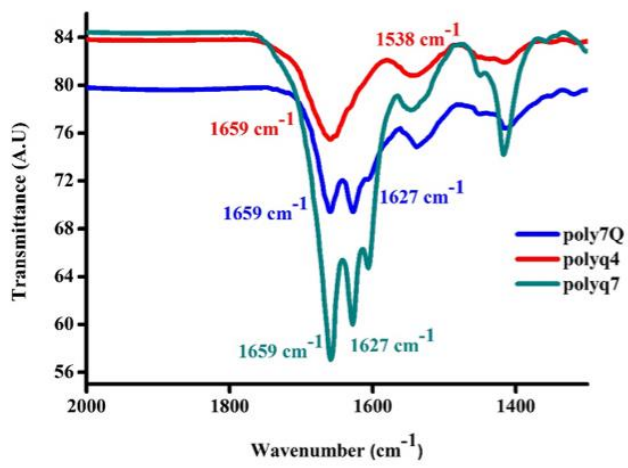

(b)

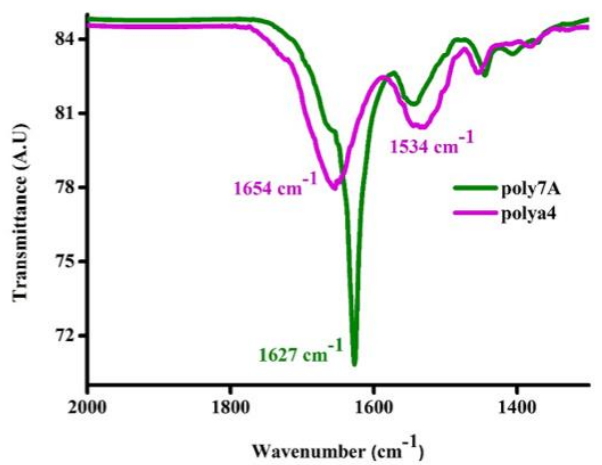

Figure S19. a. the IR spectra of Poly7Q, Polyq4, and Polyq7. b. The IR spectra of Poly7A and Polya4.

\section{REFERENCES}

1. Liu, L.; Zhang, L.; Mao, X. B.; Niu, L.; Yang, Y. L.; Wang, C. Chaperon-Mediated Single Molecular Approach toward Modulating a Beta Peptide Aggregation. Nano. Lett. 2009, 9, (12), 4066-4072. 2. Yu, L.; Zheng, Y.; Xu, J.; Qu, F.; Lin, Y.; Zou, Y.; Yang, Y.; Gras, S. L.; Wang, C. Site-Specific Determination of Ttr-Related Functional Peptides by Using Scanning Tunneling Microscopy. Nano. Res. 2018, 11, (1), 577-585.

3. Mao, X. B.; Wang, C. X.; Wu, X. K.; Ma, X. J.; Liu, L.; Zhang, L.; Niu, L.; Guo, Y. u. Y.; Li, D. H.; Yang, Y. L.; Wang, C. Beta Structure Motifs of Islet Amyloid Polypeptides Identified through Surface-Mediated Assemblies. Proc. Natl. Acad. Sci. U. S. A. 2011, 108, (49), 19605-19610. 4. Dai, H.; Wang, S.; Hisaki, I.; Nakagawa, S.; Ikenaka, N.; Deng, K.; Xiao, X.; Zeng, Q. OnSurface Self-Assembly of a C-3-Symmetric Pi-Conjugated Molecule Family Studied by Stm: TwoDimensional Nanoporous Frameworks. Chemistry-an Asian Journal 2017, 12, (19), 2558-2564. 5. Zha, B.; Miao, X.; Liu, P.; Wu, Y.; Deng, W. Concentration Dependent Halogen-Bond Density in the $2 d$ Self-Assembly of a Thienophenanthrene Derivative at the Aliphatic Acid/Graphite Interface.

Chem Commun. 2014, 50, (64), 9003-9006.

6. Nguyen Thi Ngoc, H.; Gopakumar, T. G.; Hietschold, M. Polymorphs of Trimesic Acid Controlled by Solvent Polarity and Concentration of Solute at Solid-Liquid Interface. Surf. Sci. 2013, 607, 68-73.

7. Miao, X.; Xu, L.; Cui, L.; Deng, W. Steric Matching and the Concentration Induced SelfAssembled Structural Variety of 2,7-Bis(N-Alkoxy)-9-Fluorenone at the Aliphatic Solvent/Graphite Interface. Phys Chem Chem Phys. 2014, 16, (24), 12544-12553. 